\title{
Electrospun nanofiber sorbents for the pre-concentration of urinary 1-hydroxypyrene
}

\author{
Okechukwu Clinton Ifegwu ${ }^{1,2,3}$, Chimezie Anyakora ${ }^{1,2^{*}}$, Samuel Chigome ${ }^{3}$ and Nelson Torto ${ }^{3}$
}

\begin{abstract}
Background: Synthetic polymers have some qualities that make them good candidates for pre concentration of trace analytes biological fluids because of their great potentials to be functionalized and electrospun into nanofibres.

Methods: In this study, Electrospun nanofiber sorbents fabricated from 11 polymers \{poly(styrene-co-methacrylic acid), poly(styrene-co-divinylbenzene) (SDVB), poly(styrene-co-acrylamide), poly(styrene-co-p-sodium styrene sulfonate), polystyrene, poly(vinyl benzyl chloride), cellulose acetate, polyethylene terephthalate (PET), polysulfone, nylon 6$\}$ were evaluated for the extraction and pre-concentration of 1-hydroxypyrene from a water sample.

Results: Scanning electron microscopy (SEM) studies revealed the formation of continuous fine bead-free and randomly arrayed fibers with their average diameters ranging from 110 to $650 \mathrm{~nm}$. The percentage recoveries were highest for nylon 6 with 72\%, SDVB with 70\%, whereas PET achieved the lowest recovery at 34\%. Under optimized conditions, the analyte followed a linear relationship for all sorbents in the concentration range of 1 to 1,000 $\mu \mathrm{g} / \mathrm{L}$. The coefficient of determination $\left(r^{2}\right)$ was between 0.9990 to 0.9999 , with precision (\%relative standard deviation (RSD)) $\leq 9.51 \%(n=6)$ for all the analysis. The \%RSD for intra- and inter-day precision at three different concentrations, 10, 25, and $50 \mu \mathrm{g} / \mathrm{L}$, was $\leq 7.88 \%$ for intraday and $\leq 8.04 \%$ inter-day (3 days), respectively, for all evaluated sorbents. The LOD and LOQ were found to be between 0.054 and $0.16 \mu \mathrm{g} / \mathrm{L}$ and 0.18 and $0.53 \mu \mathrm{g} / \mathrm{L}$, respectively, using a fluorescent detector.
\end{abstract}

Conclusions: The study suggested that if packed into cartridges, nylon 6 and SDVB nanofiber sorbents could serve as alternatives to the conventional C-18 sorbents in the pre-concentration and clean-up of the tumorigenic biomarker, 1-hydroxypyrene in human urine. The fabrication of selective nanofibers could also extend and simplify sample preparation for organic and biological analytes.

\section{Background}

1-Hydroxypyrene (Figure 1) is the most extensively studied biomarker of polycyclic aromatic hydrocarbons (PAHs) (Ifegwu et al. 2012; Gunier et al. 2006; Berthoin et al. 2004; Grainger et al. 2005; Van Larebeke et al. 2006). PAHs are the largest class of cancer-causing compounds and were rated the ninth world most threatening compound in 2001 (Simko 2002; King et al. 2002). Pyrene represents a significant proportion in every PAH mixture. It usually undergoes metabolism to yield 1-hydroxypyrene and conjugates that are found at trace concentration levels in complex biological matrices. The low concentrations (usually at $\mathrm{ppb}$ )

\footnotetext{
* Correspondence: canyakora@gmail.com

'Department of Pharmaceutical Chemistry, Faculty of Pharmacy, University of Lagos, Lagos, Nigeria

${ }^{2}$ The centre for Applied Research on Separation Science (CAROSS), Lagos, Nigeria Full list of author information is available at the end of the article
}

pose a challenge in the determination of 1-hydroxypyrene. For accurate determination of 1-hydroxypyrene, an efficient sample pre-concentration step is required in order to enrich 1-hydroxypyrene and bring its concentration to detectable levels by commonly used detectors such as ultraviolent/visible (UV-vis), diode array (DAD), and fluorescence (FLD).

In the past decades, liquid-liquid extraction (LLE) was the extraction technique of choice (Kataoka 2010; Oluseyi et al. 2011). It has since been overtaken by solid-phase extraction (SPE) due to its numerous demerits (Oluseyi et al. 2011; Kang et al. 2009; Kang et al. 2007; Chigome et al. 2011; Kataoka et al. 2009). SPE has gained popularity and acceptability because of its selectivity, reproducibility (Wardencki et al. 2007), ease of operation, and the wide range of available sorbent materials. However, in order to achieve higher enrichment efficiency of target analytes, the choice of suitable

\section{슬}




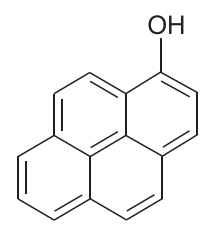

Figure 1 The chemical structure of 1-hydroxypyene.

sorbents for SPE is extremely important (Adeyemi et al. 2011). Thus, this has necessitated the search for SPE sorbents with a high surface area to volume ratio and good selectivity.

Electrospun nanofibers have been widely received due to their great analytical potential when employed as SPE sorbents (Kang et al. 2009; Kang et al. 2007; Chigome et al. 2011; Adeyemi et al. 2011). Their high specific surface areas (Liu et al. 2010; Aguado et al. 2009; Zhang et al. 2008) and ease of functionalization with various groups for specific purposes (Kang et al. 2007; Kang et al. 2008; Yoshitake et al. 2003) has increased their acceptance in the scientific community. The simplest and most flexible means of fabricating nanofibers is via electrospinning (Chigome et al. 2011; Uyar and Besenbacher 2008; Greiner and Wendorff 2007; Ramakrishna et al. 2006). The technique allows for control of the nanofibers orientation thereby influencing the fiber arrangement that has a significant effect on the performance of the subsequent sorbent material (Ma et al. 2005; Frenot and Chronakis 2003; Huang et al. 2003). Electrospinning relies on repulsive electrostatic forces to draw a viscoelastic solution into nanofibers (Chigome et al. 2011).

To the best of our knowledge, there has been no attempt to evaluate electrospun polymer nanofibers as sorbents for urinary 1-hydroxypyrene. To date, synthetic polymers are the most popular class of electrospun materials employed as SPE sorbents owing to the fact that they show the greatest potential for tuning of sorptive chemistries. Hence, in this study, 11 polymers [nylon 6, polystyrene (PST), poly(vinyl benzyl chloride) (PVBC), cellulose acetate (CA), polyethylene terephthalate (PET), polysulfone (PSO in DMF and pyridine), poly(styrene acryl amide), poly(styrene methacrylic acid), poly(styrene-co- $p$ sodium styrene sulfonate), poly(styrene-co-divinyl benzene)] were synthesized, fabricated into nanofibers, and evaluated for their efficiency in selectively extracting 1-hydroxypyrene. It was anticipated that the sorbents would interact well with 1-hydroxypyrene based on hydrophobic interactions, hydrogen bonding, van der Waal interactions or pore-filling. In addition, some of the sorbents have been reported to impart selectivities based on acidity, basicity, and polarity (Kang et al. 2009; Chigome et al. 2011; Wardencki et al. 2007; Fontanals et al. 2007; Kloskowski et al. 2009).

\section{Methods}

\section{Reagents and materials}

All chemicals were of pure analytical grade. Polystyrene $(\mathrm{Mw}=$ 192,000), tetrahydro-furan, (98.0\%), N,N-dimethylformamide (99.0\%), acetone (99.8\%), methanol, pyridine, nylon 6, cellulose acetate, polysulfone, polyethylene terephthalate, 4-vinyl benzyl chloride, styrene monomer, acryl amide, methacrylic acid, $p$-sodium styrene sulfonate, and divinyl benzene were purchased from Merck Chemicals (Wadesville, South Africa) and Sigma-Aldrich (Town, South Africa). The hydroxypyrene standard was obtained from Sigma-Aldrich (Saint Louis, MO, USA). All glassware was washed and rinsed thoroughly in ultrapure water generated from a MilliQ system, (Millipore, Billerica, MA, USA).

\section{Solutions}

A standard stock solution (1 mg/L) was prepared by dissolving an appropriate amount of 1-hydroxypyrene in few drops of methanol and made up to the expected volume with 33\% methanol. Working solutions were prepared by an appropriate dilution of the stock solutions with 33\% methanol. All solutions were stored in the fridge at $4{ }^{\circ} \mathrm{C}$.

\section{Synthesis of polymers}

Of 4-vinyl benzyl chloride, $5 \mathrm{~g}$ was transferred into a vial in which $0.04 \mathrm{~g}$ of azoisobutylnitrile (AIBN) initiator was added. It was heated under argon gas at $70^{\circ} \mathrm{C}$ for $12 \mathrm{~h}$ until polymerization was complete. The product was dissolved in $100 \mathrm{ml}$ tetrahydrofuran (THF) and transferred into $500 \mathrm{ml}$ of methanol to precipitate out the polymer. The white poly(vinyl benzyl chloride) was obtained by filtering the solution using a sintered glass connected to a suction pump and finally air dried.

Three copolymers poly(styrene-co-methacrylic acid), poly(styrene-co- $p$-sodium styrene sulfonate), and poly(styrene-co-acrylamide) were synthesized at five mole ratios of their respective co-monomers. All copolymers and homopolymers were synthesized by boiling medium emulsion polymerization. In a typical polymerization procedure, a three (with one opening blocked) or two-necked flask equipped with a reflux condenser and a magnetic bar placed in an oil bath was used. Appropriate quantities of the monomers were added to 50 to $100 \mathrm{ml}$ of water and stirred at a rotation speed of $300 \mathrm{rpm}$. The mixture was raised to reflux and after the medium had boiled for $30 \mathrm{~min}$, typically at $93^{\circ} \mathrm{C}$ to $98^{\circ} \mathrm{C}, 0.2 \mathrm{wt} . \%$ (with respect to the monomers) of the potassium persulfate initiator dissolved in an appropriate volume of water was added to the boiling medium. The reaction was left to run for $12 \mathrm{~h}$ to ensure complete polymerization before water removal by a rotary evaporator and subsequent oven or air-drying of the resultant polymer powders.

For poly(styrene-co-divinyl benzene) (SDVB), the initiator 2,2' -azobisisobutyrnitrile was dissolved in the monomers (S 
and DVB in a 25:1 molar ratio) and the diluents (toluene + water) after which the suspension was boiled for $24 \mathrm{~h}$. The product was extracted in THF and washed in methanol and water.

\section{Fabrication of nanofibers}

Appropriate amount of the readily available polymers and synthesized polymers were dissolved in suitable solvents to give $12 \%$ cellulose acetate, $20 \%$ polystyrene, $30 \%$ polyethylene terephthalate, $16 \%$ nylon, two sets of $20 \% \mathrm{w} / \mathrm{v}$ polysulfone dissolved in pyridine and dimethylformamide (DMF), 18 wt.\% each of poly(styrene-co-methacrylic acid), 20 wt.\% each of poly(styrene-co- $p$-sodium styrene sulfonate), and poly(styrene-co-acrylamide), 33 wt.\% poly(vinyl benzyl chloride), $50 \mathrm{wt}$ \% poly(styrene-co-divinyl benzene). These viscoelastic solutions were then electrospun to obtain continuous fine nanofibers which were employed in the sorption studies of 1-hydroxypyrene.

In the setup, a viscoelastic polymer solution was loaded into a polypropylene $(25 \mathrm{ml})$ syringe. A 21 gauge, $90^{\circ}$ blunt end stainless steel needle of internal diameter $0.8 \mathrm{~mm}$ was connected directly to the luer tip of the syringe. A high electric field is generated between the viscoelastic polymer solution contained in a syringe and a metallic collection plate by connecting the needle of the syringe to a high voltage power supply. At a sufficient high frequency where the repulsive electrostatic force overcomes the surface tension of the polymer solution, a droplet draws out into a coneshaped terminus and sprays downwards towards the flat plate collector (aluminum foil). As the jet travels towards the collector plate, the solvents dry off and the jet deposits as a mesh of nanofibers on the collector. The electrospinning setup consists of three basic components: a high voltage power supply, a mode to deliver a viscoelastic solution, and a means of collecting the fibers. All polymer solutions were driven by a syringe pump with a consistent flow rate.

\section{Characterization of morphology}

The morphologies of the nanofibers were studied with the aid of a Vegan Tescan (TS5136ML) scanning electron microscope (Brno, Czech Republic) operating at an accelerated voltage of $20 \mathrm{kV}$ after gold sputter coating. The fibers were peeled in thin sheets and placed on the surface of the gold coating before introducing it into the SEM machine. The copolymers were characterized using Fourier transform infrared (FTIR). The fibers were placed on the sample compartment of the FTIR, the knob was adjusted to make contact with the fiber and beam splitter, and the characteristic peaks were thereafter detected and displayed on the screen.

\section{Adsorption studies}

The adsorption of 1-hydroxypyrene by 11 nanofibers was investigated. Of the various electrospun nanofibers, $10 \mathrm{mg}$ was added to vials containing aliquots of 1-hydroxypyrene of different concentrations while varying the volume and contact time. The concentrations, volume, and times under investigation were 1.0 to $100 \mu \mathrm{g} \mathrm{L}-1,2 \mathrm{~mL}$, and 10 to $60 \mathrm{~min}$, respectively. The solutions were shaken using a shaker, filtered, and the fibers were dried under a gentle flow of nitrogen for $2 \mathrm{~min}$, prior to desorption studies. The concentration of 1-hydroxypyrene remaining in the solution was then determined using high-performance liquid chromatography with postcolumn fluorescence derivatization (HPLC-FLD). The amount adsorbed by the fibers was calculated from the difference between the initial and final concentration of solutions. Six replicates of each solution were prepared.

\section{Desorption studies}

1-Hydroxypyrene adsorbed on the fibers was desorbed with $1 \mathrm{ml}$ of $100 \%$ methanol and was made up to the mark for all samples. The eluate was then analyzed with HPLC-FLD.

\section{Chromatographic procedure}

The HPLC analysis was carried out using an Agilent 1200 system equipped with FLD and autosampler (Agilent Technologies, Inc., Santa Clara, CA, USA). The stationary phase was an X-Bridge octadecyl $(150 \mathrm{~mm} \times 4.6 \mathrm{~mm})$ $5 \mu \mathrm{m}$. Aqueous mobile phase $(0.5 \%$ phosphoric acid) and methanol $(10: 90, v / v)$ were used. The wavelengths of excitation and emission were 254 and $400 \mathrm{~nm}$, respectively, with the flow rate at $0.8 \mathrm{~mL} / \mathrm{min}$. All measurements were performed at $37^{\circ} \mathrm{C}$ temperature, and a Chem. station HPLC software package (Agilent Technologies, Inc., Santa Clara, CA, USA) was used for the data analysis.

\section{Validation of the assay}

\section{Linearity of calibration curve}

Standard stock solutions were diluted with 33\% methanol to produce concentrations of 1 to $1,000 \mu \mathrm{g} / \mathrm{L}$ of 1-hydroxypyrene. A calibration plot was constructed using the 1-hydroxypyrene peak area against the various concentrations $(1,10,50,100,500,1,000 \mu \mathrm{g} / \mathrm{L})$, and the linear regression equation alongside correlation coefficient was evaluated.

\section{Accuracy and precision}

Recoveries were determined as percentages of the measured desorption concentrations against the initial concentrations for 1-hydroxypyrene solutions at $10 \mu \mathrm{g} / \mathrm{L}(n=$ 3 for each). The intra-day and inter-day precision and accuracy were evaluated for 10,25 , and $50 \mu \mathrm{g} / \mathrm{L}$ hydroxypyrene standards on the same day and on three consecutive days after adsorption on the fibers and desorption with methanol $(n=6)$. Imprecision was reported as the relative standard deviation (RSD) of analyte concentration. 


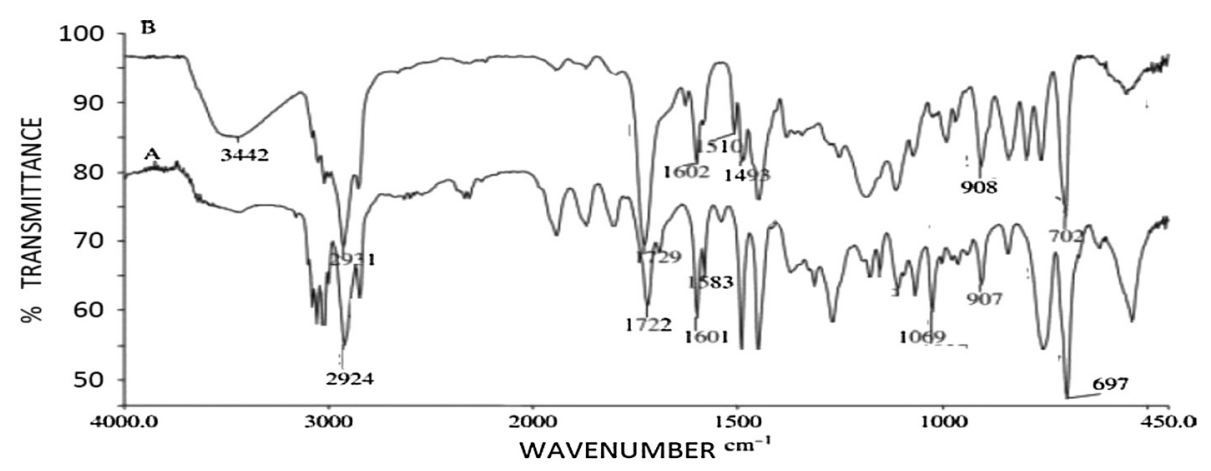

Figure 2 FTIR images of polystyrene (A) and poly (styrene-co-divinyl benzene) (B).

\section{Limit of detection and quantification}

The limit of detection (LOD) and limit of quantification (LOQ) were evaluated on the basis of signal-to-noise ratio of 3:1 and 10:1, respectively.

\section{Results and discussion \\ FTIR spectra}

The characteristic peaks at 702 and $908 \mathrm{~cm}^{-1}$ on SDVB were used to assign substitutions on aromatic rings (Figure 2). The peaks were similar to 697 and $907 \mathrm{~cm}^{-1}$ on the PST, and $1,069 \mathrm{~cm}^{-1}$ was due to aryl $-\mathrm{H}$ in plane bands. The peaks observed at 1,510,1,602, and $1,729 \mathrm{~cm}^{-1}$ were from the $\mathrm{C}=\mathrm{C}$ stretching bands for the aromatic rings, and the peak around 2,931 was assigned to $\mathrm{C}-\mathrm{H}$ stretching for $\mathrm{Sp}^{2}$ carbon. The broad peak at $3,442 \mathrm{~cm}^{-1}$ may be attributed to $-\mathrm{OH}$ stretch from the residual water molecule.

The FTIR spectra of polystyrene copolymers synthesized from various mole ratios ranging from 0:1 (styrene: $p$-sodium styrene sulfonate) to 1:0 (styrene: $p$-sodium styrene sulfonate) are shown in Figure 3 . The broad peak at $3,450 \mathrm{~cm}^{-1}$ is due to the $\mathrm{O}-\mathrm{H}$ stretching vibration of residual water. The peak becomes stronger and sharper with increasing $p$-sodium styrene sulfonate in the feed indicating its incorporation onto the polystyrene backbone. The presence of the characteristic peaks of sulfonate groups (around $1,041 \mathrm{~cm}^{-1}$ representing symmetric stretching vibration of the $\mathrm{SO}_{3}$ groups and $1,182 \mathrm{~cm}^{-1}$ representing asymmetric stretching vibration of the $\mathrm{SO}_{3}$ groups) at the mole ratios 10:1 to 20:1 confirmed the successful incorporation of the sulfonate functionality onto the polystyrene backbone.

A characteristic peak around $1,698 \mathrm{~cm}^{-1}$ in Figure 4 is attributed to the carbonyl stretching vibration of the carboxyl groups in the methacrylic acid component of the polymers. With the increase in the methacrylic acid content in the feed, the intensity of the peak was observed to gradually increase which provided confirmatory evidence of the successful incorporation of the carboxyl group onto the polystyrene backbone. In addition, the well-defined peaks around $2,924 \mathrm{~cm}^{-1}\left(-\mathrm{CH}_{2}\right.$ asymmetric stretching) and $3,026 \mathrm{~cm}^{-1}$ (aromatic $\mathrm{CH}$ stretching) from a mole ratio of 5:1 confirmed the predominance of the polystyrene

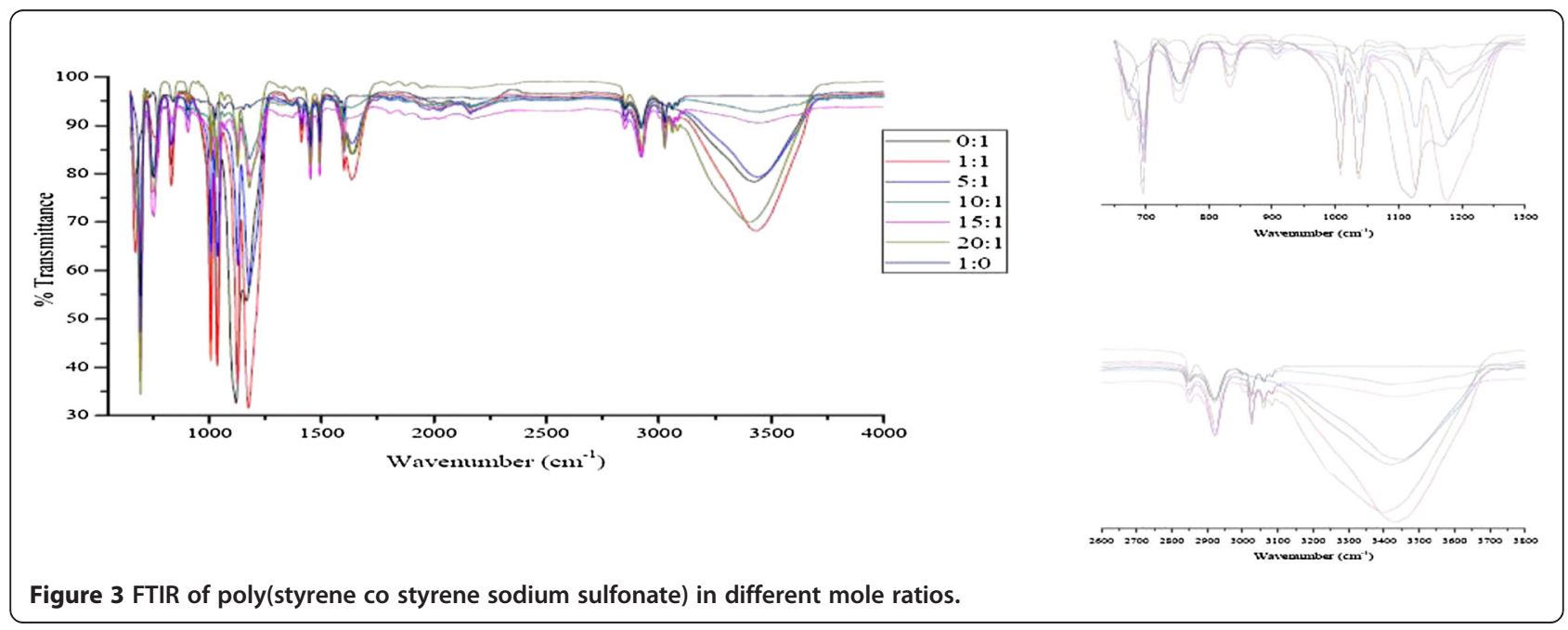




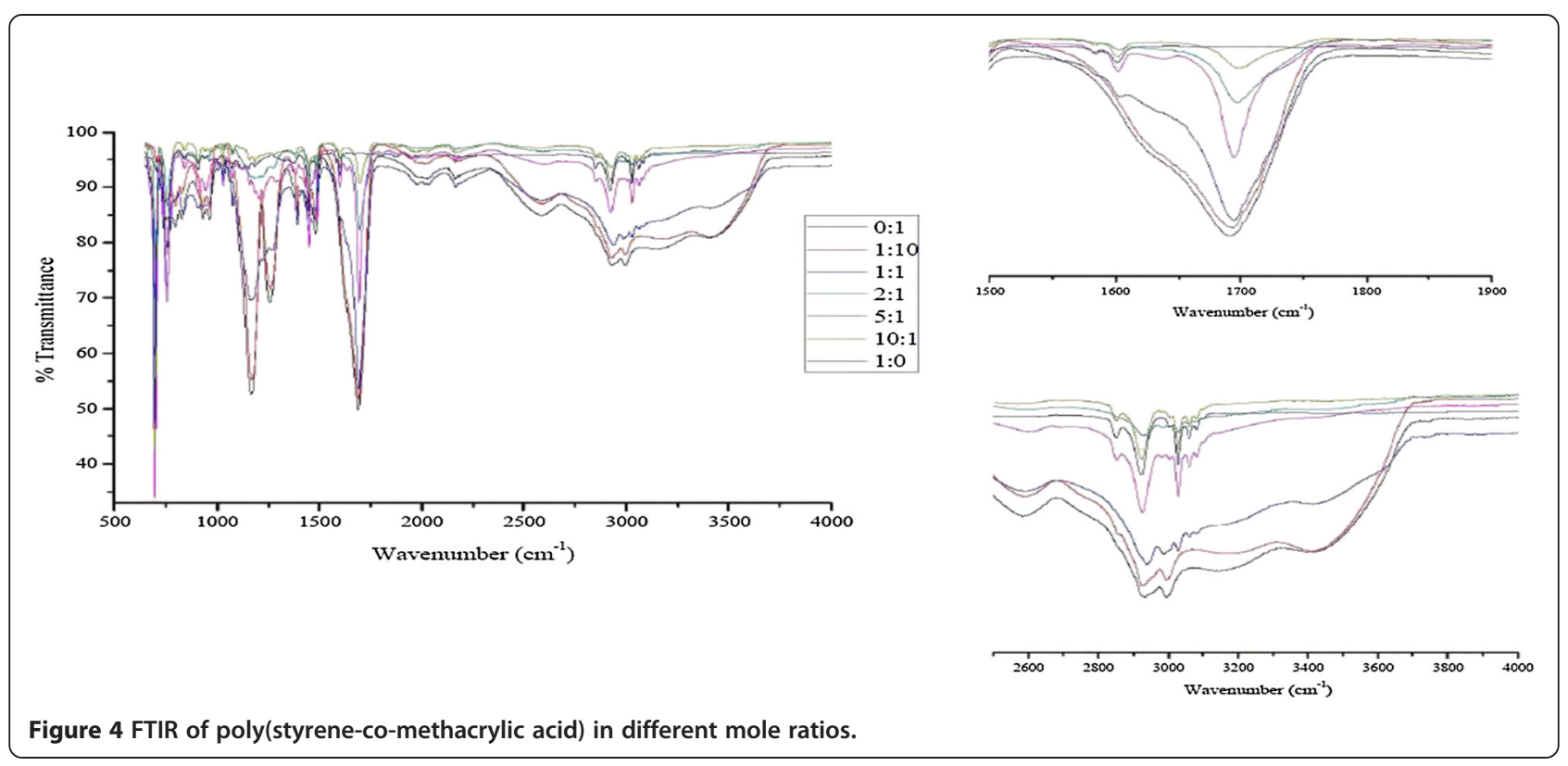

backbone. The presence of the carbonyl stretch at the mole ratio 10:1 confirmed the incorporation of the carboxylic acid functionality onto the polystyrene backbone.

Peaks around $3,200 \mathrm{~cm}^{-1}$ (N-H stretching), $1,650 \mathrm{~cm}^{-1}$ ( $\mathrm{C}=\mathrm{O}$ stretching), 1,420, and $1,105 \mathrm{~cm}^{-1}$ all characteristic of the acrylamide monomer were observed in Figure 5. With the increase in the acrylamide content in the feed, the intensity of the peak at $1,650 \mathrm{~cm}^{-1}$ was observed to gradually increase which provided confirmatory evidence of the successful incorporation of the amide group onto the polystyrene backbone. The presence of the carbonyl stretch at the mole ratio 10:1 confirmed the incorporation of the acrylamide functionality onto the polystyrene backbone.

\section{Morphology of electrospun nanofibers}

The SEM images of 11 nanofibers that are shown in Figure 6 illustrate that the nanofibers were all continuous fine bead-free and randomly arrayed. However, their sizes were remarkably different with their average diameters ranging between 110 and $650 \mathrm{~nm}$. A thinner fiber diameter is presumed to increase sorption efficiency of adsorbents. Electrospinning solvents also played major roles in the fiber morphologies and their resulting percentage recoveries. This is evident from the higher sorption recovery displayed by polysulfone in DMF when compared to polysulfone in pyridine as shown in Table 1 . The high conductivity of DMF might have favored the formation of smooth fibers of smaller diameter. Similarly,

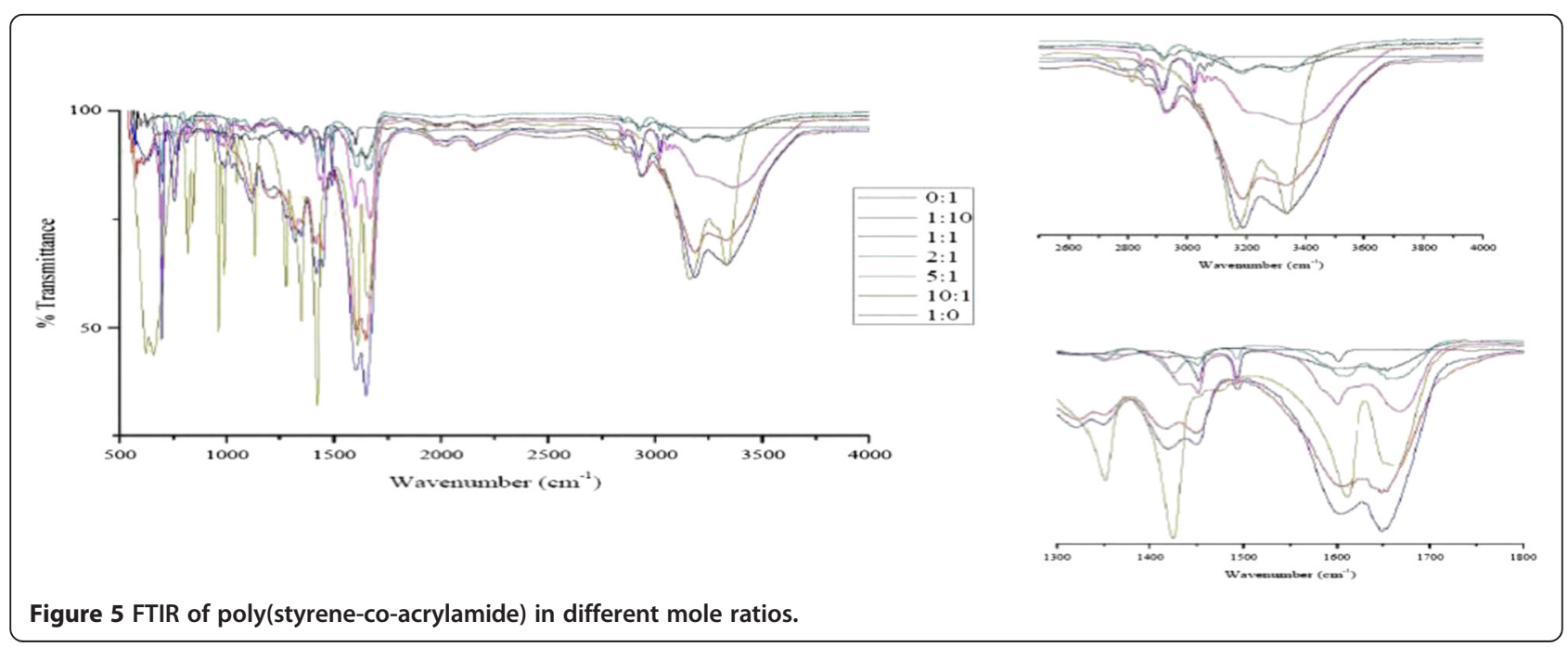




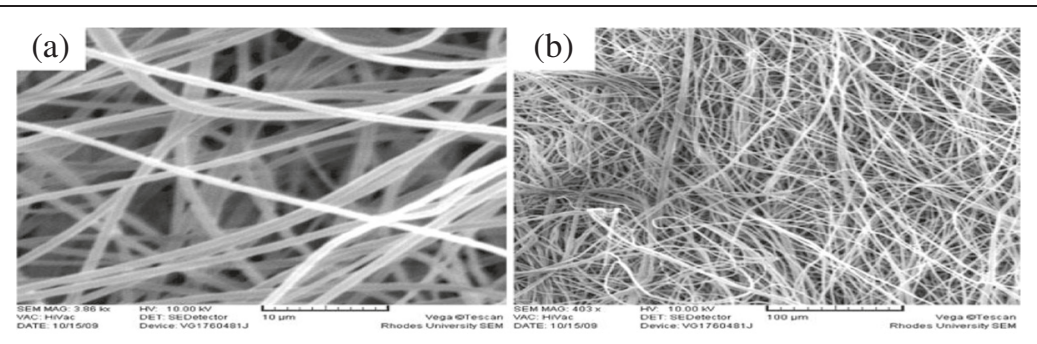

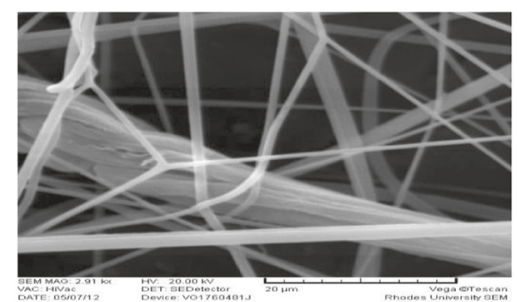

(c)

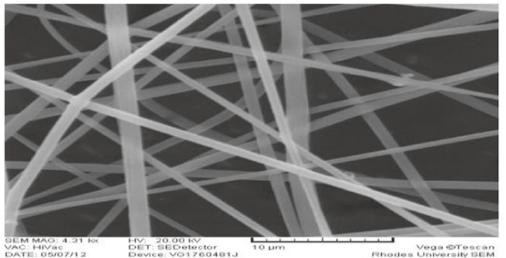

(e)

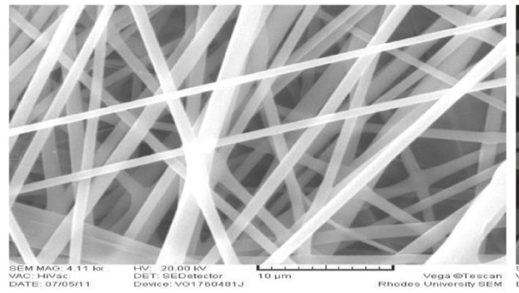

(g)

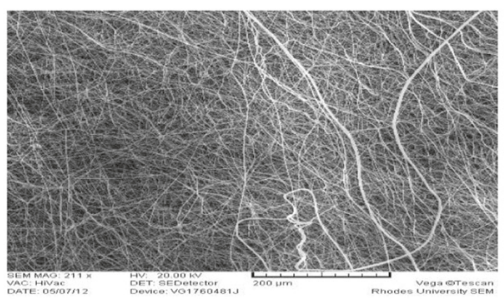

(i)

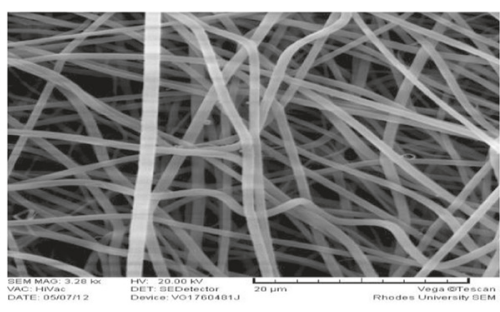

(d)

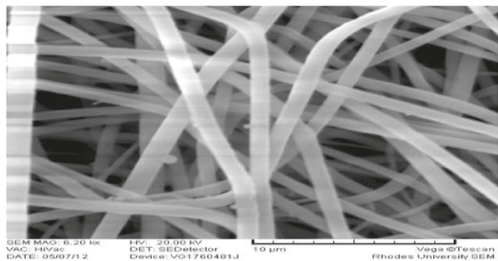

(f)

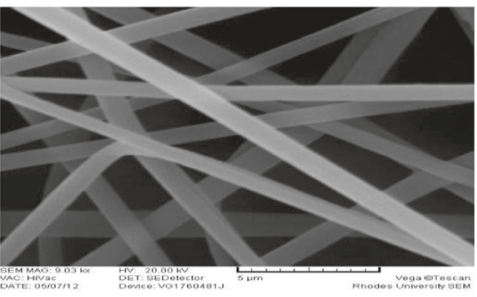

(h)

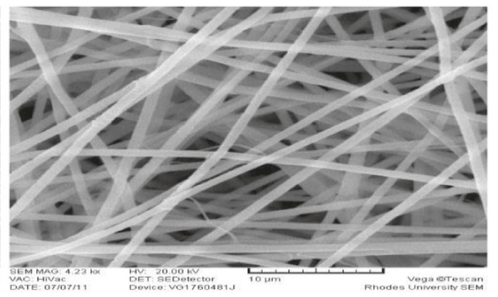

(j)

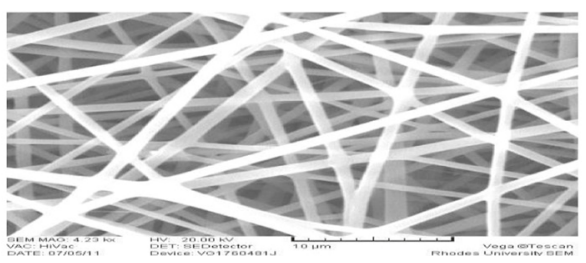

(k)

Figure 6 SEM images. Polystyrene (a), CA (b), SDVB (c), PVBC (d), PSO/DMF (e), PSO/Py (f), ST/NaST.SO 3 (g), PET (h), nylon 6 (i), ST/Methacrylic acid (j), and ST/Acrylamide (k). 
Table 1 Analytical parameters for the preconcentration of 1-hydroxypyrene

\begin{tabular}{|c|c|c|c|c|c|c|c|c|c|c|c|c|}
\hline \multirow{2}{*}{$\begin{array}{l}\text { Electrospun } \\
\text { nanofibers }\end{array}$} & \multirow{2}{*}{$\begin{array}{l}\text { Linear } \\
\text { range/ppb }\end{array}$} & \multirow[t]{2}{*}{ Linearity, $r^{2}$} & \multicolumn{4}{|c|}{ Repeatability (\%RSD) } & \multirow[t]{2}{*}{ LOD/ppb } & \multirow[t]{2}{*}{ LOQ/ppb } & \multicolumn{4}{|c|}{ Average $\%$ recovery } \\
\hline & & & $10 \mathrm{ppb}$ & $25 \mathrm{ppb}$ & $50 \mathrm{ppb}$ & $100 \mathrm{ppb}$ & & & $10 \mathrm{ppb}$ & $25 \mathrm{ppb}$ & $50 \mathrm{ppb}$ & $100 \mathrm{ppb}$ \\
\hline PSO/PYR & 1 to 1,000 & 0.9993 & 3.63 & 5.38 & 7.19 & 7.83 & 0.121 & 0.403 & 56 & 48 & 47 & 45 \\
\hline PSO/DMF & 1 to 1,000 & 0.9999 & 2.75 & 3.61 & 5.83 & 7.19 & 0.094 & 0.311 & 64 & 57 & 57 & 54 \\
\hline PET & 1 to 1,000 & 0.9990 & 4.15 & 8.11 & 6.47 & 9.51 & 0.085 & 0.283 & 38 & 38 & 38 & 34 \\
\hline CA & 1 to 1,000 & 0.9994 & 3.78 & 5.46 & 7.88 & 8.23 & 0.054 & 0.181 & 49 & 48 & 46 & 40 \\
\hline PST & 1 to 1,000 & 0.9996 & 3.75 & 6.23 & 6.89 & 7.65 & 0.096 & 0.320 & 52 & 51 & 49 & 43 \\
\hline Nylon 6 & 1 to 1,000 & 0.9999 & 2.72 & 3.93 & 5.27 & 7.11 & 0.087 & 0.291 & 72 & 70 & 69 & 64 \\
\hline PVBC & 1 to 1,000 & 0.9998 & 4.21 & 8.67 & 8.61 & 8.43 & 0.160 & 0.533 & 50 & 50 & 47 & 43 \\
\hline SDVB & 1 to 1,000 & 0.9997 & 3.25 & 5.23 & 6.55 & 8.04 & 0.079 & 0.263 & 70 & 68 & 67 & 63 \\
\hline ST/A.Amide & 1 to 1,000 & 0.9990 & 4.33 & 7.41 & 6.74 & 9.11 & 0.115 & 0.383 & 65 & 63 & 62 & 58 \\
\hline ST/M.Acid & 1 to 1,000 & 0.9999 & 2.83 & 4.16 & 5.24 & 7.27 & 0.075 & 0.251 & 68 & 65 & 65 & 60 \\
\hline $\mathrm{ST} /{\mathrm{S} . \mathrm{SO}_{3}}_{3}$ & 1 to 1,000 & 0.9996 & 3.91 & 5.22 & 7.83 & 6.39 & 0.086 & 0.287 & 61 & 60 & 58 & 55 \\
\hline
\end{tabular}

fine continuous nanofibers of polystyrene were spun by combining DMF:THF (4:1). The study has shown that the higher conductivity of DMF and volatility of THF favored the formation of smooth nanofibers (Uyar and Besenbacher 2008; Uyar et al. 2009; Xu et al. 2010a; Xu et al. 2010b).

\section{Sorption studies}

Figure 7 shows the effect of contact time between the sorbent and 1-hydroxypyrene. The results indicate that all sorption processes were mostly achieved within the first $30 \mathrm{~min}$ of reaction. The process became more gradual until equilibrium was reached after $1 \mathrm{~h}$. At this point, the adsorbent was saturated with the analyte. While some of the sorbent reached their maximum adsorption capacity at $25 \mathrm{~min}$, others were at $35 \mathrm{~min}$. This means that the contact time is an important parameter in selecting a suitable sorbent. Sorbent with higher hydrophilic functional groups would be expected to adsorb the analyte faster due to more effective sorbent-analyte interaction resulting

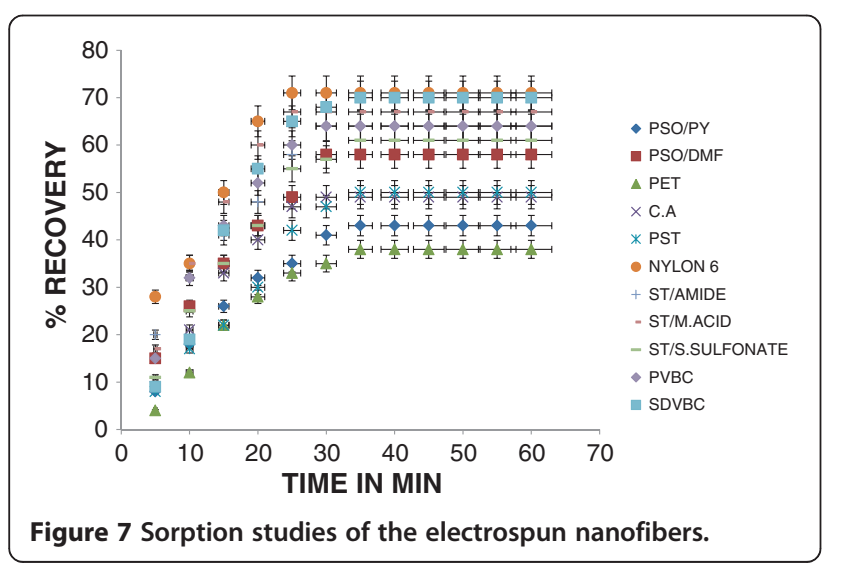

from increase in mass transfer (Xu et al. 2010a; Xu et al. 2010b; Qi et al. 2008; Bagheri et al. 2012). This suggests that the hydrophilic functional groups like amide and carboxylic groups on some of the sorbents did contribute greatly to the rapid sorption of 1-hydroxypyrene. Such fast adsorption kinetics was an added advantage of the sorbent as it allows for a high throughput of samples prior to analysis.

\section{Adsorption and desorption studies}

Figure 8 shows the amount of analyte that was desorbed from the nanofibers. The highest and lowest percentage adsorption level obtained from this study were 93\% and $48 \%$ for poly(vinyl benzyl chloride) and polyethylene terephthalate, respectively, while $34 \%$ and $72 \%$ represented the lowest and highest desorption values for PET and nylon 6 , respectively. While the percentage adsorption was fairly constant with increasing hydroxypyrene concentration, there was a gradual decrease in percentage desorbed, thus increasing the difference between the percentage adsorption and percentage desorption for all 11 fibers. It is noteworthy that the huge difference between the adsorption (96\%) and desorption (43\%) of hydroxypyrene in PVBC strongly suggests a feasible chemical reaction between the $-\mathrm{OH}$ group of the analyte and the $-\mathrm{CH}_{2}-\mathrm{Cl}$ of the PVBC owing to the fact that the halogen groups like chlorine are a good leaving group.

\section{Choice of nanofiber/adsorbent}

Figure 9 shows the percentage recovery profile for all 11 fibers at varying concentrations. While nylon 6 gave a high recovery of $72 \%$, PET gave the lowest recovery of $38 \%$. The performance of nanofibers as SPE sorbents is largely dependent on their surface characteristics, morphology, composition, and the polymer functionalities (Kang et al. 2009; Kang et al. 2007; Kataoka et al. 2009). The 


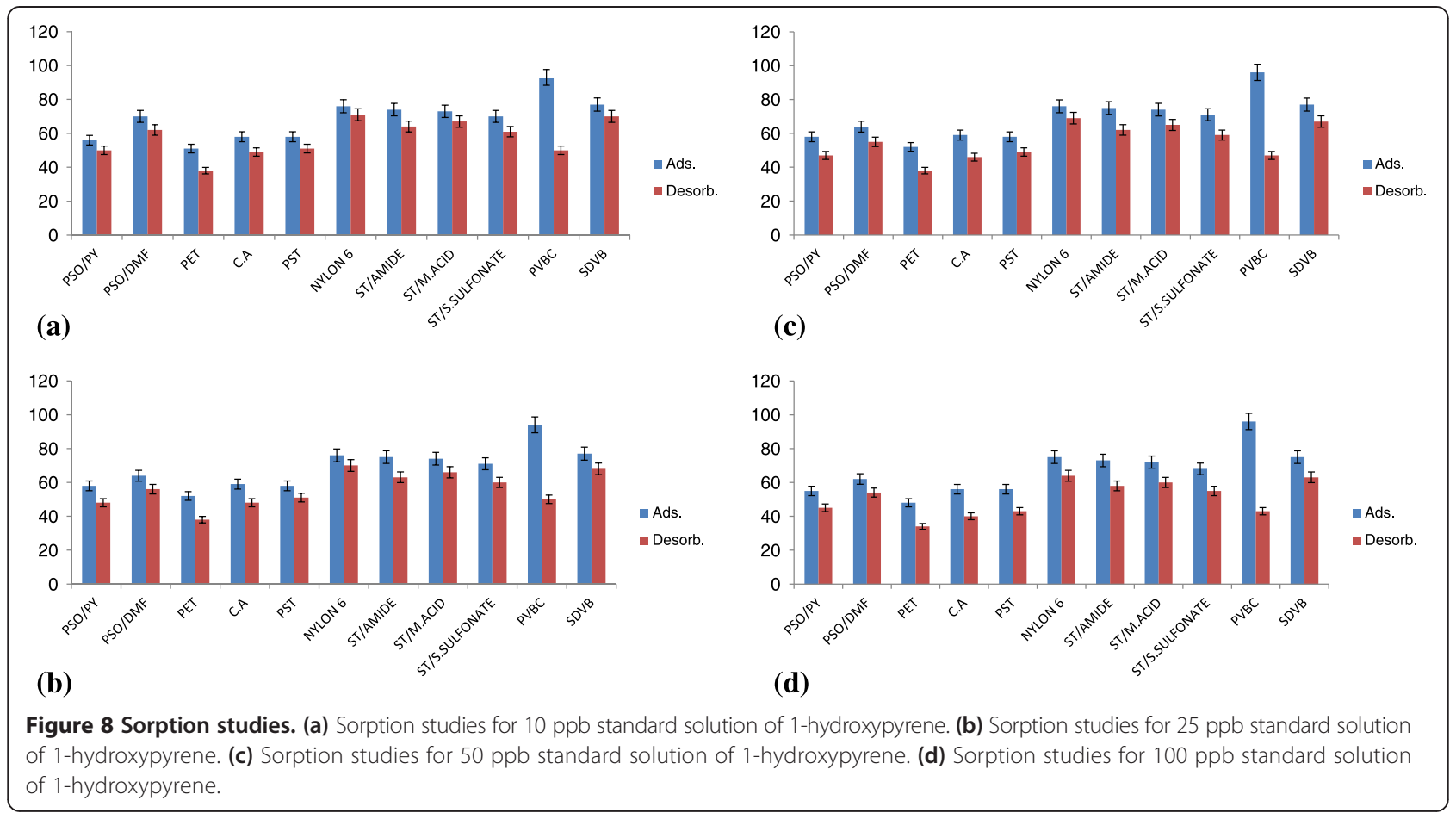

experimental results revealed that the presumed hydrophobicity of the nanofibers and hydrogen bonding exhibited significant effects on their sorption rate and capacity towards 1-hydroxypyrene. The good recovery of nylon 6 could possibly be due to the amphiphilic characteristics of nylon 6. It is also an indication that chemical interactions were possibly involved in the sorption processes. First, the amide groups on nylon 6 makes them hydrophilic, thus improving nanofibers' wettability resulting in a strong adsorption affinity for the target analyte (Xu et al. 2010a, b). There is also the possibility of hydrogen bonding between the amide group of nylon 6 and the $-\mathrm{OH}$ group on the surface of the analyte with any of the two acting as hydrogen bonding donor. Moreover, the role of the strong hydrophobic interaction between the $\pi$-electrons of the methylene groups of nylon 6 and the $\pi$-electrons of the poly aromatic hyroxypyrene cannot be overemphasized (Kloskowski et al. 2009; Xu et al. 2010a; Xu et al. 2010b).

More so, the aromatic rings of the matrix network on SDVB permits the electron-donor interactions between the sorbent and the bonds of the aromatic analyte. This further increases the analyte-sorbent interaction (Bagheri et al. 2012; Marce and Borrull 2000) which is a likely reason for its high sorption recovery of slightly over $70 \%$ as well. Furthermore, the - $\mathrm{OH}$ groups on the surface of the analyte can act as a hydrogen-bonding donor and form hydrogen bonds with organic molecules (Marce and Borrull 2000), indicating that hydrogen bonding interactions could as well be playing a role in the SDB

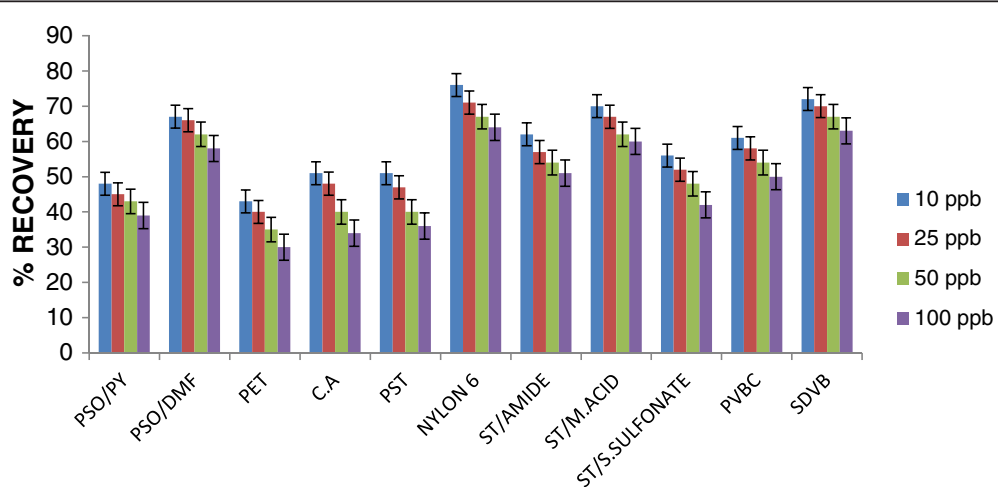

Figure 9 Percentage recoveries of 1-hydoxypyrene at different concentrations. 
sorption system. Despite the large numbers of potential binding sites and relatively higher hydrophobicity in unfunctionalized polystyrene nanofibers, the functionalized polymers and co-polymers showed better extraction efficiency. Theoretically, the amide group introduced in poly(styrene-co-acrylamide) and the carboxyl group on poly(styrene-co-methacrylic acid) will enhance the water movement into the sorbent, improving mass transfer and making the sorbent-analyte interaction more effective (Liu et al. 2010; Kloskowski et al. 2009; Qi et al. 2008).

Pore-filling is equally a viable sorption mechanism that could be playing a significant role in adsorption. Even though the adsorption mechanisms of these nanofibers are relatively complicated, the probable chemistries involved are $\pi-\pi$ bonding interaction, hydrogen bonding, pore-filling, and to a very low extent van der Waal forces.

Due to the aforementioned chemistries and qualities of these fibers, some researchers have also explored the feasibility of using poly(styrene-co-methacrylic acid), poly (styrene-co-pstyrene sulfonate), polystyrene, and nylon $6\left(-\left[\mathrm{NH}-\left(\mathrm{CH}_{2}\right)\right.\right.$ 5-CO]n-) electrospun nanofiber mats as SPE sorbents to directly extract trace organic pollutants from environmental water (Chigome et al. 2011; Liu et al. 2010; Fontanals et al. 2007; Kloskowski et al. 2009; Qi et al. 2008).

\section{Analytical parameters}

Table 1 profiles the analytical parameters including the percentage recoveries of sorbents. The HPLC-FLD analytical method employed in assessing the nanofiber sorbents was found to be linear in the concentration range of 1 to $1,000 \mu \mathrm{g} / \mathrm{L}$. The correlation coefficient $\left(r^{2}\right)$ ranged between 0.9990 and 0.9999 , with relative standard deviation $(\mathrm{RSD}) \leq 9.51 \%(n=6)$ for all the analysis. The \%RSD for intra- and inter-day precision at three different concentrations, 10,25 , and $50 \mu \mathrm{g} / \mathrm{L}$, were $\leq 7.88 \%$ for intraday and $\leq 8.04 \%$ inter-day ( 3 days) for all the sorbent assessed. The LOD and LOQ were found to be between 0.054 and 0.16 and 0.18 and 0.53 , respectively.

\section{Conclusions}

Eleven electrospun nanofibers were successfully fabricated and evaluated as potential sorbents for the preconcentration of 1-hydroxypyrene, a PAH biomarker. In this study, nylon 6 and poly(styrene-co-divinylbenzene) were found to be the most favorable sorbents for 1hydroxypyrene in terms of their sorption capacity and percentage recoveries. Their percentage recoveries were $72 \%$ and $70 \%$, respectively. However, we believe that these recoveries could be improved if these nanofibers are packed in cartridges or discs as sorbent bed. Because of their nanoscale diameters, controllable surface configurations, various polymeric compositions, coupled with sorbent tenability for easy desorption, electrospun nanofibers are potential sorbents in the enrichment of 1hydroxypyrene and provide a significant improvement over conventional SPE sorbents.

\section{Competing interests}

The authors declare that they have no competing interests.

\section{Authors' contributions}

CA, NT and OCl designed the experiment. The experiment was carried out by $\mathrm{OCl}$ and SC. The manuscript was written by $\mathrm{OCl}$ and CA. All authors read and approved the final manuscript.

\section{Author details}

'Department of Pharmaceutical Chemistry, Faculty of Pharmacy, University of Lagos, Lagos, Nigeria. ${ }^{2}$ The centre for Applied Research on Separation Science (CAROSS), Lagos, Nigeria. ${ }^{3}$ Department of Chemistry, Rhodes University, Grahamstown, South Africa.

Received: 26 January 2014 Accepted: 12 March 2015 Published online: 08 April 2015

\section{References}

Adeyemi D, Mokgadi J, Darkwa J, Anyakora C, Ukpo G, Turner C, Torto N (2011) Electrospun nanofibers sorbents for preconcentration of 1,1-dichloro-2,2 bis-(4-chlorophenyl)ethylene with subsequent desorption by pressurized hot water extraction. Chromatographia 73:1015-1020

Aguado I, Arsuaga JM, Arencibia A, Lindo M, Gascon V (2009) Aqueous heavy metals removal by adsorption on amine-functionalized mesoporous silica. J Haz Mater 163:213-221

Bagheri H, Ayazi Z, Aghakhani A, Alipour N (2012) Polypyrrole/polyamide electrospun-based sorbent for microextraction in packed syringe of organophosphorous pesticides from aquatic samples. J Sep Sci 35:114-120

Berthoin KK, Broeckaert FF, Robin MM, Haufroid W, Burbure CD, Bernard A (2004) Serum pneumoproteins and biomarkers of exposure to urban air pollution: a cross-sectional comparison of policemen and foresters. Biomarkers 9(4-5):341-352

Chigome S, Darko G, Torto N (2011) Electrospun nanofibers as sorbent material for solid phase extraction. Analyst 136:2879-2889

Fontanals N, Marce RM, Borull F (2007) New materials in sorptive extraction techniques for polar compounds. J Chromatographia A 1152:14-31

Frenot A, Chronakis IS (2003) Polymer nanofibers assembled by electrospinning. Curr Opin Colloid Interface Sci 8:64

Grainger JJ, Huang WW, Li ZZ, Edwards SS, Walcott CC, Smith CC, Turner WE, Wang RR, Patterson DD (2005) Polycyclic aromatic compounds. Taylor Francis Ltd 25:47

Greiner A, Wendorff JH (2007) Electrospinning: a fascinating method for the preparation of ultrathin fibers. Angewandte Chemie Int Edition 46(30):5670-5703

Gunier RB, Reynolds P, Hurley SE, Yerabati S, Hertz A, Strickland P (2006) Estimating exposure to polycyclic aromatic hydrocarbons: a comparison of survey, biological monitoring, and geographic information system-based methods. Cancer Epidemiol Biomarkers Prev 15:1376-81

Huang ZM, Zhang YZ, Kotaki M, Ramakrishna S (2003) A review on polymer nanofibers by electrospinning and their applications in nanocomposites. Compos Sci Technol 63:2223-2253

Ifegwu C, Osunjaye K, Fashogbon F, Oke K, Adeniyi A, Anyakora C (2012) Urinary 1-hydroxypyrene as a biomarker to carcinogenic polycyclic aromatic hydrocarbon. Expo Biomarkers Can 4:7-17

Kang X, Pan C, Xu Q, Yao Y, Wang Y, Qi D, Gu Z (2007) The investigation of electrospun polymer nanofibers as a solid-phase extraction sorbent for the determination of trazodone in human plasma. Anal Chim Acta 587:75-81

Kang X, Chen L, Zhang Y, Liu Y, Gu Z (2008) Performance of electrospun nanofibers for SPE of drugs from aqueous solutions. J Sep Sci 31:3272-3278

Kang XJ, Chen LQ, Wang Y, Zhang YY, Gu ZZ (2009) Design of packed-fiber solid-phase extraction device for analysis of the drug and its metabolite in plasma. Biomed Microdevices 11:723-729

Kataoka H (2010) Recent developments and applications of microextraction techniques in drug analysis. Anal Bioanal Chem 396:339-364 
Kataoka H, Ishizaki A, Nonaka Y, Saito K (2009) Developments and applications of capillary microextraction techniques: a review. Anal Chim Acta 655:8-29

King SS, Meyer JS, Andrews AR (2002) Polycyclic aromatic hydrocarbons in soil using hollow fiber membrane solvent microextraction. J Chromatogr A 982:201-208

Kloskowski A, Pilarczyk M, Przyjazny A, Namiesnik J (2009) Progress in development of molecularly imprinted polymers as sorbents for sample preparation. Crit Rev Anal Chem 39:43-58

Liu Z, Kang X, Fang F (2010) Solid phase extraction with electrospun nanofibers for determination of retinol and a-tocopherol in plasma. Microchim Acta 168:59-64

Ma Z, Kotaki M, Ramakrishna S (2005) Electrospun cellulose nanofiber as affinity membrane. J Membr Sci 265:115-123

Marce RM, Borrull F (2000) Solid-phase extraction of polycyclic aromatic compounds. J Chromatogr A 885:273-290

Oluseyi T, Olayinka K, Alo B, Smith RM (2011) Comparison of extraction and clean-up techniques for the determination of polycyclic aromatic hydrocarbons in contaminated soil samples. J Environ Scie Technol 5(7):482-493

Qi D, Kang X, Chen L, Zhang Y, Wei H, Gu Z (2008) Electrospun polymer nanofibers as a solid-phase extraction sorbent for the determination of trace pollutants in environmental water. Anal Bioanal Chem 390:929

Ramakrishna S, Fujihara K, Teo W-E, Yong T, MA Z, Ramaseshan R (2006) Electrospun nanofibers: solving global issues. Materials Today 9(3):40-50

Simko PP (2002) Determination of polycyclic aromatic hydrocarbons in smoked meat products and smoke flavouring food additives. Chromatogr J P 770:3-18

Uyar T, Besenbacher F (2008) Electrospinning of uniform polystyrene fibers: the effect of solvent conductivity. Polymer 49:5336-5343

Uyar T, Havelund R, Nur Y, Hacaloglu J, Besenbacher F, Kingshott P (2009) Molecular filters based on cyclodextrin functionalized electrospun fibers. J Membr Sci 332:129-137

Van Larebeke NA, Bracke ME, Nelen W, Koppen GG, Schoeters GA, Van Loon HH, Vlietinck RR (2006) Differences in tumor-associated protein levels among middle-age Flemish women in association with area of residence and exposure to pollutants. Environ Health Perspect 114:887-892

Wardencki W, Curylo J, Namiesnik J (2007) Trends in solventless sample preparation techniques for environmental analysis. J Biochem Biophys Methods 70:275-288

Xu Q, Wu S-Y, Wang M, Yin X-Y, Wen Z-Y, Ge W-N, Gu Z-Z (2010a) Electrospun nylon6 for the extraction of three estrogens in environmental water samples. Chromatographia 71:487-492

Xu Q, Yin X, Wu S, Wang M, Wen Z, Gu Z (2010b) Determination of phthalate esters in water samples using Nylon6 nanofibers mat-based solid-phase extraction coupled to liquid chromatography. Microchim Acta 168:267-275

Yoshitake H, Yokoi T, Tatsumi T (2003) Adsorption behavior of arsenate at transition metal cations captured by amino-functionalized mesoporous silica. Chem Mater 15:1713-1721

Zhang Y, Kang X, Chen L, Pan C, Yao Y, Gu Z-Z (2008) Fiber-packed SPE tips based on electrospun fibers. Anal Bioanal Chem 391:2189-2197

\section{Submit your manuscript to a SpringerOpen ${ }^{\circ}$ journal and benefit from:}

- Convenient online submission

- Rigorous peer review

- Immediate publication on acceptance

- Open access: articles freely available online

- High visibility within the field

- Retaining the copyright to your article

Submit your next manuscript at springeropen.com 